\title{
Application of the Shifted Frequency Internal Equivalence to Two Dimensional Lossy Objects
}

\author{
Buğra Aydemir ${ }^{1}$, Adnan Köksal ${ }^{2}$ \\ ${ }^{1,2}$ Electrical and Electronics Engineering Department, Hacettepe University, Turkey \\ ${ }^{1}$ corresponding author, E-mail: aydemirbugra@gmail.com
}

\begin{abstract}
Performance of a new method, Shifted Frequency Internal Equivalence (SFIE) is studied in the analysis of scattering from two dimensional lossy objects. This method finds the solution of a wideband scattering problem faster than classical approaches.
\end{abstract}

By introducing conductivity, loss shows itself as imaginary electrical permittivity. Changing conductivity changes the distribution of electromagnetic waves and modifies Radar Cross Section (RCS) plots. In this study homogeneous and inhomogeneous conductive media are investigated to widen the usage of SFIE method. By increasing conductivity skin effect phenomenon is observed, electrical dimensions are decreased and RCS plots are flattened as expected.

Numerical results obtained by SFIE are compared to the ones obtained by Method of Moments and the differences are shown.

\section{Introduction}

The demands of numerical electromagnetic analysis of scattering objects increase in proportion to the developments in radar, antennas and microwave technologies etc. Solution algorithms for wideband problems with Method of Moments (MoM) and Finite Element Method (FEM) require filling impedance matrix and solving it for new frequencies [1], [2]. Since integral calculations are done for every single frequency, several methods are introduced to speed up this process such as; interpolation, asymptotic approach and characteristic basis function method [3]-[5]. All these methods rely on approximating the new MoM matrix and therefore are different from our approach where the method is exact at the new frequency. Shifted Frequency Internal Equivalence method reuses volume elements and calculates a part of surface elements only [6]. The approximate behavior results from the discretization involved in the numerical solution. Since volume elements are reused with some basic algebraic manipulations instead of taking all the integrals, SFIE decreases the calculation time significantly. The number of recalculated surface elements is much less than number of volume elements typically; therefore there is a big reduction in computation cost [7], [8].

Until now, the studies related to SFIE were dealing with ideal and lossless cases. Since all materials are lossy and waves behave differently in conductive and non-conductive media, testing SFIE for lossy scatterers is needed to increase the applicability of the method. In this study, only electric loss due to conductivity is taken into account, magnetic loss is ignored.

Firstly, effect of conductivity is demonstrated on objects with constant electrical dimensions and skin effect is displayed. Then, this effect is shown on objects with constant real dimensions. Next, inhomogeneous media are studied. Finally, wideband solutions for RCS are given for objects with constant conductivity.

MoM formulation for volume integral equations is chosen to be the reference method and the numerical results given by SFIE and MoM are compared. RCS comparisons with respect to observation angle and frequency are provided. In addition to comparison of RCS results, $\mathbf{z}$ component of the internal electric field, $\mathbf{E}_{\mathbf{Z}}$, is compared for various conductivities, frequencies and sizes.

\section{Theory}

Consider an arbitrary two dimensional scatterer object with electromagnetic parameters given as $\varepsilon$ and $\mu$. Incident fields are known and shown by $\mathbf{E}_{\boldsymbol{\omega}}^{\mathbf{i}}$ and $\mathbf{H}_{\boldsymbol{\omega}}^{\mathbf{i}}$ as in Figure 1.

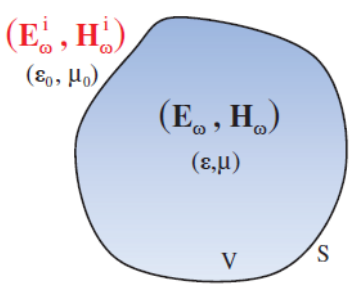

Figure 1: Original Problem

In Figure 1, $\mathrm{V}$ and $\mathrm{S}$ stand for volume and surface respectively. In two dimensional structures $\mathrm{V}$ and $\mathrm{S}$ are converted to area and closed line, but $\mathrm{V}$ and $\mathrm{S}$ are kept in this paper to make the analysis general.

According to the SFIE principle this structure is represented in free space with electrical and magnetic sources in $\mathrm{V}$ and on $\mathrm{S}$ as in Figure 2. 


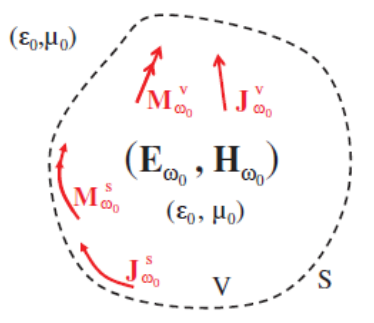

Figure 2: Internal Equivalence Principle

In a similar manner, external equivalence without scatterer is shown in Figure 3.

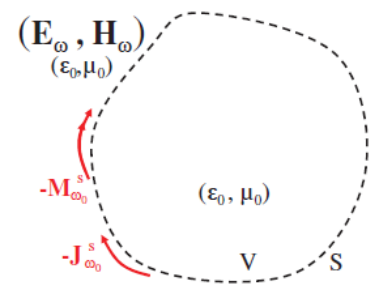

Figure 3: External Equivalence Principle

Since tangential fields on surface are continuous, internal and external equivalence equations are written and matched at the boundary. The internal equivalence parts taken from these integral equations are calculated just once by using SFIE since sources radiate at $\omega_{0}$.

Incident fields in free space satisfy a set of Maxwell's equations. A second set of equations is found in scatterer with medium parameters. Total fields in $\mathrm{V}$ and on $\mathrm{S}$ are calculated using SFIE with the following sources.

$$
\begin{aligned}
\mathbf{J}_{\omega_{0}}^{V} & =\mathrm{j}\left(\omega \varepsilon-\omega_{0} \varepsilon_{0}\right) \mathbf{E}_{\omega} \\
\mathbf{M}_{\omega_{0}}^{V} & =\mathrm{j}\left(\omega \mu-\omega_{0} \mu_{0}\right) \mathbf{H}_{\omega} \\
\mathbf{J}_{\omega_{0}}^{S} & =-\hat{n} \times \mathbf{H}_{\omega} \\
\mathbf{M}_{\omega_{0}}^{S} & =\hat{n} \times \mathbf{E}_{\omega}
\end{aligned}
$$

Normal vector $\hat{n}$ is defined outwards of the surface. To apply external equivalence, $-\mathbf{J}_{\omega_{0}}^{S}$ and $-\mathbf{M}_{\omega_{0}}^{S}$ sources are used and the following equations are found.

$$
\begin{gathered}
\mathbf{E}_{r \omega_{0}}\left(\mathbf{J}_{\omega_{0}}^{v}, \mathbf{M}_{\omega_{0}}^{v}, \mathbf{J}_{\omega_{0}}^{s}, \mathbf{M}_{\omega_{0}}^{s}\right)=\mathbf{E}_{\omega} \\
\mathbf{H}_{r \omega_{0}}\left(\mathbf{J}_{\omega_{0}}^{v}, \mathbf{M}_{\omega_{0}}^{v}, \mathbf{J}_{\omega_{0}}^{s}, \mathbf{M}_{\omega_{0}}^{s}\right)=\mathbf{H}_{\omega} \\
\hat{n} \times\left[\mathbf{E}_{r \omega_{0}}\left(\mathbf{J}_{\omega_{0}}^{v}, \mathbf{M}_{\omega_{0}}^{v}, \mathbf{J}_{\omega_{0}}^{s}, \mathbf{M}_{\omega_{0}}^{s}\right)\right] \times \hat{n}= \\
\hat{n} \times\left[\mathbf{E}_{r \omega}\left(-\mathbf{J}_{\omega_{0}}^{s},-\mathbf{M}_{\omega_{0}}^{s}\right)+\mathbf{E}_{\omega}^{i}\right] \times \hat{n} \\
\hat{n} \times\left[\mathbf{H}_{r \omega_{0}}\left(\mathbf{J}_{\omega_{0}}^{v}, \mathbf{M}_{\omega_{0}}^{v}, \mathbf{J}_{\omega_{0}}^{s}, \mathbf{M}_{\omega_{0}}^{s}\right)\right] \times \hat{n}= \\
\hat{n} \times\left[\mathbf{H}_{r \omega}\left(-\mathbf{J}_{\omega_{0}}^{s},-\mathbf{M}_{\omega_{0}}^{s}\right)+\mathbf{H}_{\omega}^{i}\right] \times \hat{n}
\end{gathered}
$$

Equation (2.1) and (2.2) are volume equivalences. Equation (2.3) and (2.4) match internal and external electric and magnetic tangential fields [9].
To apply method of moments the geometry is discretized. For two dimensional problems, triangles are frequently used for the discretization. Then, basis functions are employed for expansion. In TM or TE cases there are 3 unknowns. This study assumes $\mathrm{TM}_{Z}$ case, therefore every triangle has $\mathbf{E}_{Z}$, $\mathbf{H}_{x}, \mathbf{H}_{y}$ unknown fields. Surface unknowns can also be defined if used. The surface currents in this study require 2 unknowns as $\mathbf{J}_{s}, \mathbf{M}_{s}$ for outer side of every triangle at the boundary. As a result, there are $3 m+2 p$ unknowns in total, where $\mathrm{m}$ and $\mathrm{p}$ stand for volume and surface triangles respectively. Traditional MoM formulation for volume integral equations requires $3 \mathrm{~m}$ unknowns.

Finally integral equations are tested for every volume and surface unknown using point matching. The impedance matrix is a square matrix with $3 m+2 p$ columns and rows. The unknowns related to volume elements are calculated just once for $\omega_{0}$ and reused with some basic algebraic manipulations for other frequencies. As it can be seen in Equation (3) surface interactions are calculated for every single frequency. The structure of impedance matrix, unknowns and excitation vector are given in Equation (3).

$$
\left[\begin{array}{cc}
Z_{\mathrm{VV}}\left(\omega_{0}\right) & Z_{\mathrm{VS}}\left(\omega_{0}\right) \\
Z_{\mathrm{SV}}\left(\omega_{0}\right) & Z_{\mathrm{SS}}\left(\omega_{0}\right)+Z_{\mathrm{SS}}(\omega)
\end{array}\right]\left[\begin{array}{l}
X_{\mathrm{V}} \\
X_{\mathrm{S}}
\end{array}\right]=[\mathrm{V}(\omega)]
$$

$\mathrm{V}$ and S subscripts stand for volume and surface interactions respectively. $\mathrm{X}_{\mathrm{V}}$ and $\mathrm{X}_{\mathrm{S}}$ are unknowns and $\mathrm{V}$ is the excitation vector [10].

The steps for SFIE algorithm are given as;

1- Set the initial frequency $\omega_{0}$.

2- Calculate matrix elements which depend on $\omega_{0}$.

3- Set $\omega$ and calculate incident fields.

4- Calculate matrix elements which depend on $\omega$.

5- Multiply matrix elements found in step 2 with required constants.

6- Find matrix equation and solve for total fields.

7- Go back to step 3 for a new frequency solution.

Loss is added as conductivity constant and the equations are written for lossy case. Conductivity creates a current which can be written as $\mathbf{J}=\sigma \mathbf{E}$ and this current changes the Ampere's equation as,

$$
\begin{aligned}
\nabla \times \mathbf{H} & =\mathbf{j} \omega \mathbf{D}+\mathbf{J} \\
& =\mathrm{j} \omega \varepsilon \mathbf{E}+\sigma \mathbf{E} \\
& =\mathrm{j} \omega\left(\varepsilon-\mathrm{j} \frac{\sigma}{\omega}\right) \mathbf{E}
\end{aligned}
$$

The imaginary part in this equation is responsible for total loss. Loss tangent, which is defined as $\tan \delta=\sigma / \omega \varepsilon$ is often given as a criterion to show how lossy a medium is. In microwave frequencies materials are generally defined with their real electric permittivity, $\varepsilon^{\prime}=\varepsilon_{r} \varepsilon_{0}$, and the tangent loss for given frequency. That is why loss can be taken into account by using complex permittivity as $\varepsilon=\varepsilon^{\prime}-\mathrm{j} \varepsilon^{\prime \prime}$ instead of a real constant [11]. 
Changes on permittivity require wave equation to be altered as following,

$$
\nabla^{2} \mathbf{E}+\omega^{2} \mu \varepsilon\left(1-\mathrm{j} \frac{\sigma}{\omega \varepsilon}\right) \mathbf{E}=0
$$

Thus wave number becomes, $k^{2}=\omega^{2} \mu \varepsilon[1-\mathrm{j}(\sigma / \omega \varepsilon)]$ and propagation constant can be written as,

$$
\gamma=\alpha+\mathrm{j} \beta=\mathrm{j} \omega \sqrt{\mu \varepsilon} \sqrt{1-\mathrm{j}(\sigma / \omega \varepsilon)}
$$

Explicit forms of attenuation $(\alpha)$ and phase $(\beta)$ constants are given in Equations (7.1) and (7.2) respectively [12].

$$
\begin{aligned}
& \alpha=\omega \sqrt{\frac{\mu \varepsilon}{2}\left[\sqrt{1+\left[\frac{\sigma}{\omega \varepsilon}\right]^{2}}-1\right]} \\
& \beta=\omega \sqrt{\frac{\mu \varepsilon}{2}\left[\sqrt{1+\left[\frac{\sigma}{\omega \varepsilon}\right]^{2}}+1\right]}
\end{aligned}
$$

SFIE method is modified according to the changes in constants and equations as explained above.

\section{Numerical Results}

\subsection{Algorithm Comparison}

Since loss is introduced via conductivity, SFIE formulation for lossless case must give exactly the same result as SFIE formulation for lossy case with $\sigma=0$. As an example a circular scatterer at an arbitrary frequency is chosen and RCS results are checked. As expected SFIE algorithm for lossless case gives exactly the same results with lossy SFIE algorithm with $\sigma=0$. This is shown in Figure 4 .

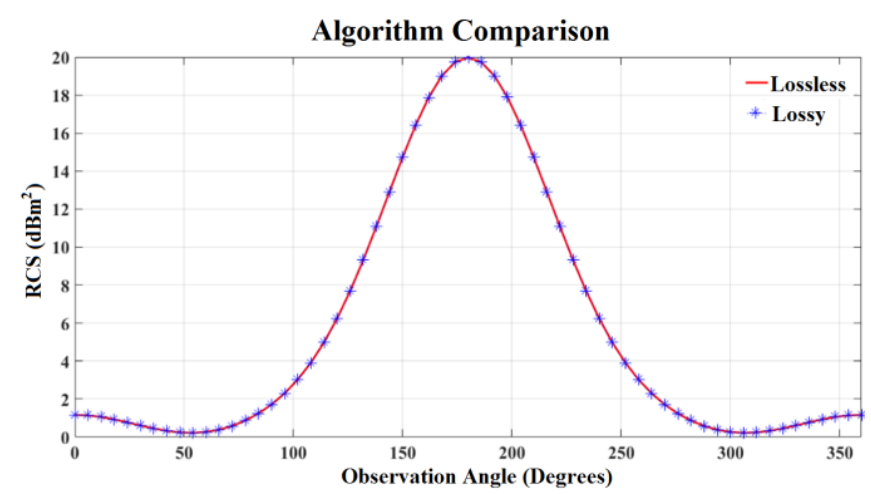

Figure 4: Comparison of SFIE algorithm for lossless and lossy cases with $\sigma=0$.

\subsection{Effect of conductivity on a scatterer with constant electrical dimensions}

Physically speaking, by increasing $\sigma$ the scatterer becomes more conductive. This changes the fields on scatterer and
RCS plots. Because wavelength in scatterer is found via $\lambda=$ $2 \pi / \beta$ and $\beta$ depends on $\sigma$, electrical dimensions of scatterer depend on conductivity. Since it is aimed to investigate the effect of conductivity only, all the other parameters are needed to be kept constant. This is why for every conductivity value, the real dimensions of the scatterer are recalculated as given in Figure 5.

Consider a plane wave at $1 \mathrm{GHz}$ which is sent in -x direction, where the coordinate system is as shown in Figure 8. This coordinate system is valid for all the structures in the paper. A circular scatterer with parameters $\varepsilon^{\prime}$ and $\mu^{\prime}$ of 2 , radius of $0.5 \lambda$ is discretized with 1052 triangles. Conductivity is increased gradually and field distribution on scatterer is observed with SFIE method. The field distribution results for increasing $\sigma$ values are given in Figure 5 (a-h).

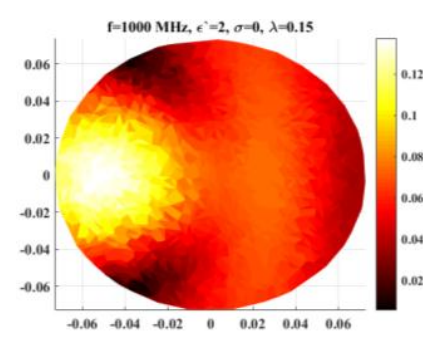

(a)

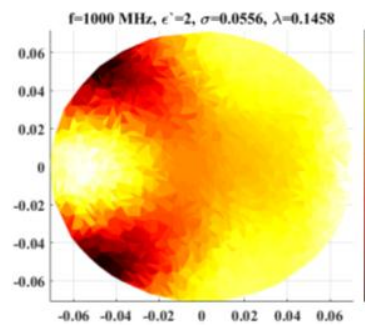

(c)

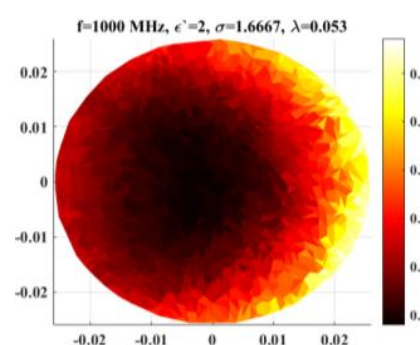

(e)

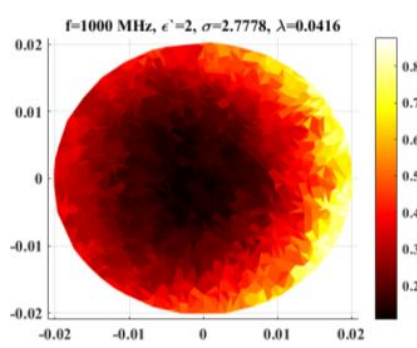

(g)

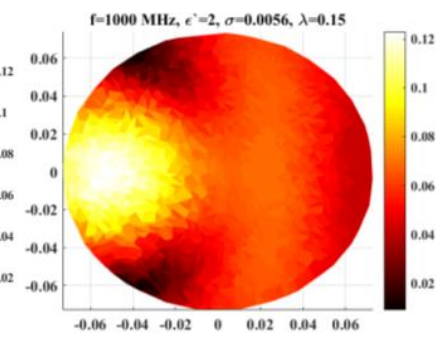

(b)

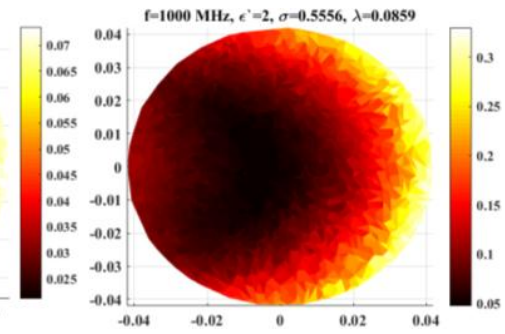

(d)

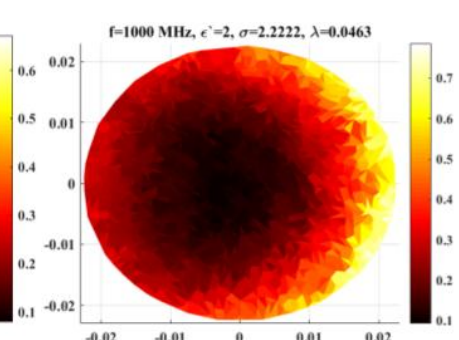

(f)

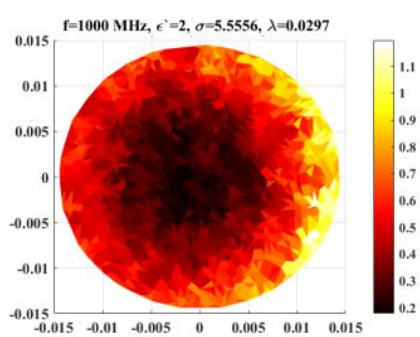

(h)
Figure 5: Field distribution as $\sigma$ increases (a) $\sigma=0$, (b) $\sigma=0.0056$, (c) $\sigma=0.0556$, (d) $\sigma=0.5556$, (e) $\sigma=1.6667$, (f) $\sigma=2.2222,(\mathrm{~g}) \sigma=2.7778$, (h) $\sigma=5.5556$ 
Wavelength decreases with $\sigma$, so real radius is decreased to keep the electrical length the same. The color scale is kept automatic to be able to see the distribution of fields clearly. The increase in the difference between upper and lower limits of color scale is consistent with skin effect phenomenon.

In addition to concentration of fields near the surface due to the skin effect, RCS plots also start to flatten. As $\sigma$ increases real part of electric permittivity starts to be negligible with respect to the imaginary part and scattering happens equally as from a PEC body.

This behavior is shown with SFIE method in Figure 6. A circular scatterer with $1 \lambda$ radius is discretized with 1988 triangles and it is illuminated with an incident field at $1 \mathrm{GHz}$.

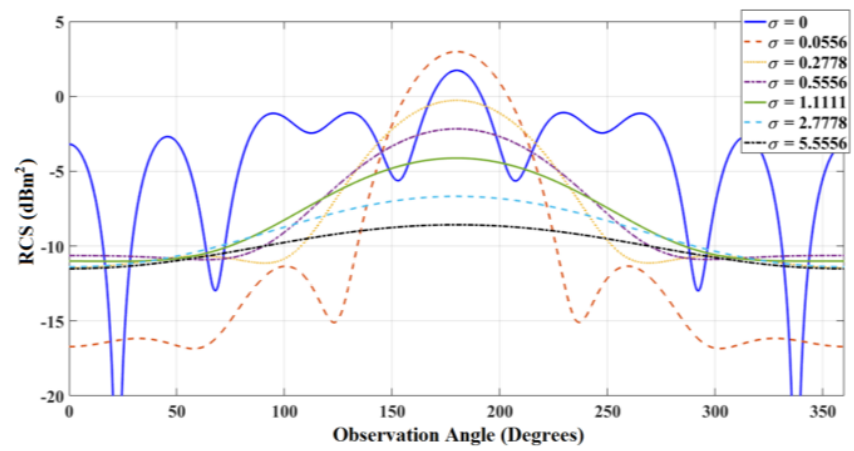

Figure 6: Effect of $\sigma$ on RCS

The ripples that occur for the lossless case diminish when the scatterer becomes lossy and the fields accumulate around the surface [13]. The structure loses its dielectric scatterer properties as shown above.

\subsection{Effect of conductivity on a scatterer with constant real dimensions}

Skin effect is also seen when the real dimensions are kept constant and electrical dimensions change. As an example, a circular homogeneous scatterer with $15 \mathrm{~cm}$ radius is illuminated at $1 \mathrm{GHz}$. Electric permittivity and magnetic permeability constants are chosen to be 2 . Field distribution for different conductivity values from 0 to 5.5556 are given in Figure 7. Corresponding wavelength values are given in the same figure so that the growth in electrical size can be seen clearly.

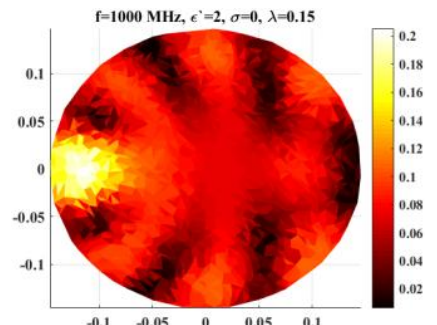

(a)

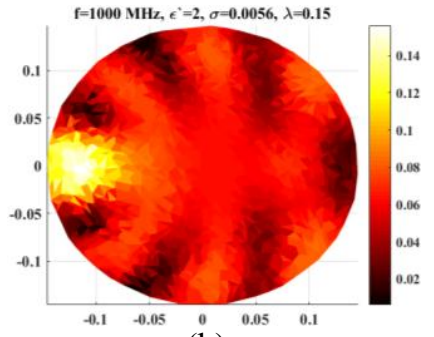

(b)

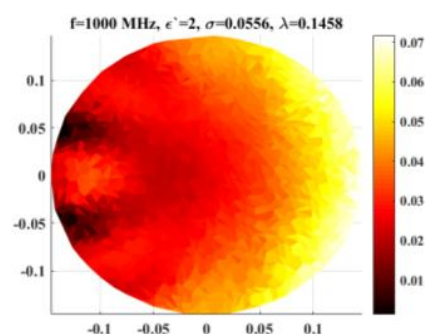

(c)

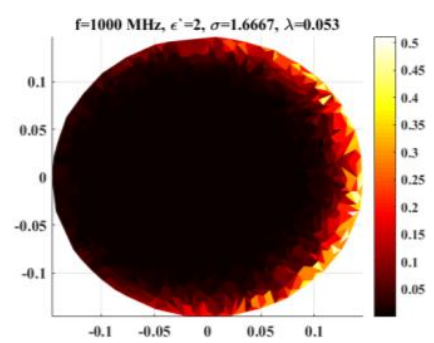

(e)

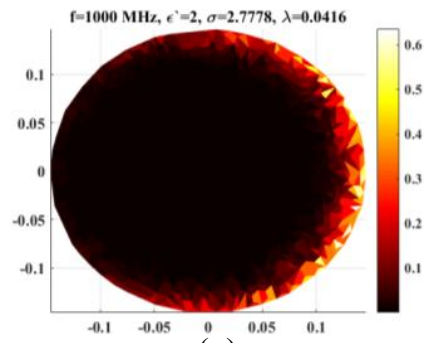

(g)

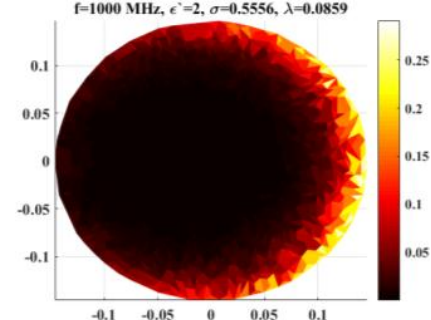

(d)

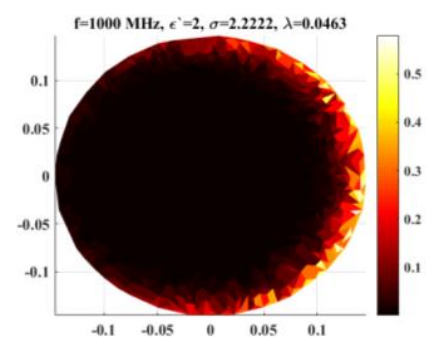

(f)

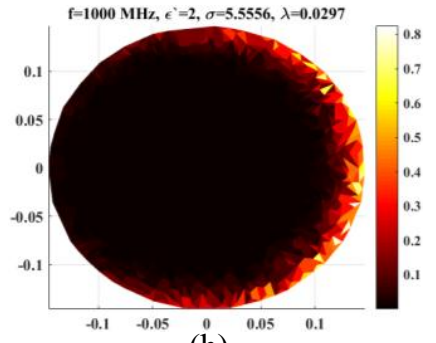

(h)
Figure 7: Distribution of fields on a scatterer with constant real dimensions as $\sigma$ increases. (a) $\sigma=0$, (b) $\sigma=0.0056$, (c) $\sigma=0.0556, \quad$ (d) $\sigma=0.5556, \quad$ (e) $\sigma=1.6667$, (f) $\sigma=2.2222$, (g) $\sigma=2.7778$, (h) $\sigma=5.5556$

Skin effect is seen in Figure 7. Electrical length of the scatterer in (h) is approximately 5 times larger than the one in (a). If the field values for different conductivities are subtracted from each other and the color axis is set constant, skin effect can be observed more clearly.

\subsection{Effect of conductivity on an inhomogeneous square scatterer with constant real dimensions}

Since SFIE uses volume integral equations, inhomogeneous scatterers can also be analyzed with this method. Figure 8 is an example for this kind of structures. A square scatterer with 3 equal sub pieces is chosen. Total length is $0.25 \lambda_{0}$ and real part of the permittivity constants are given as 10 for outer pieces and 2 for the one in the middle. Permeability is 2 throughout the structure. The scatterer is discretized with 2060 triangles. Since permittivity constant is a measure of the capability of a medium to store energy, it is expected that the outer pieces would have larger field strength in lossless case. However, increasing $\sigma$ causes imaginary part of the complex permittivity constant to increase as well. After some point the real part would be negligible and the difference between pieces becomes insignificant. This can be observed via field distribution in the scatterer. 


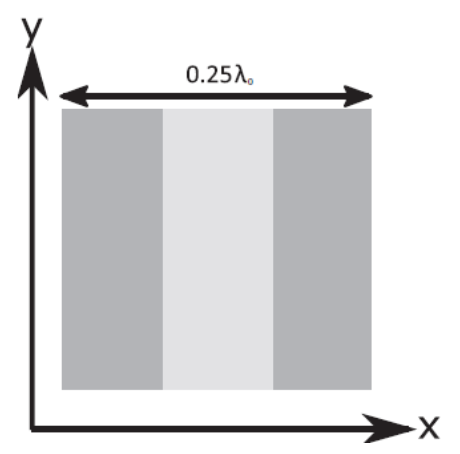

Figure 8: Inhomogeneous square problem

Wavelength in homogeneous structures is constant everywhere in the scatterer. However, an inhomogeneous structure as shown above causes wavelength to change at boundaries between pieces. Higher permittivity and conductivity lead to smaller wavelength, that is why the number of triangles to discretize the structure is chosen to be large. Real dimensions are kept constant.

Field distribution in inhomogeneous scatterer is analyzed for two different cases. In the first case, this scatterer is illuminated by an incident wave coming in $-\mathbf{x}$ direction and in the second case the incident field comes at $+45^{\circ}$ from $-\mathbf{x}$ direction.

In contrast to circular scatterers, the field distribution from a square scatterer is expected to show edge diffraction phenomenon. It is foreseen that using more accurate expansion functions would show this effect more clearly. This study which uses step function for expansion, however, shows diffraction to some extent.

The case where the incident field is sent in diagonal direction has no symmetry because the corner of the square has different distances to the middle piece on different sides of the diagonal.

To see the effect of $\sigma$ in inhomogeneous square scatterer, two cases are shown in Figure 9 and 10. In both cases the conductivity values are set as (a) $\sigma=0$, (b) $\sigma=0.0056$, (c) $\sigma=0.0278$ (d) $\sigma=0.0556$, (e) $\sigma=0.2778$, (f) $\sigma=0.5556$.

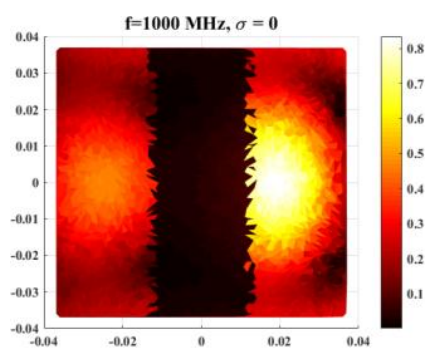

(a)

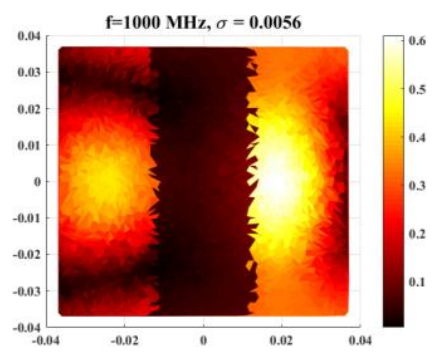

(b)

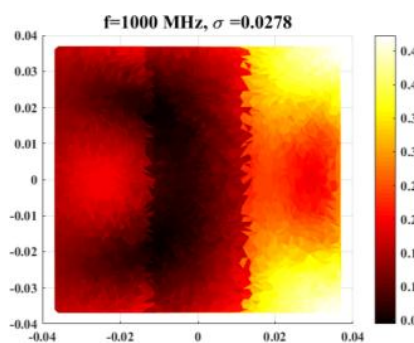

(c)

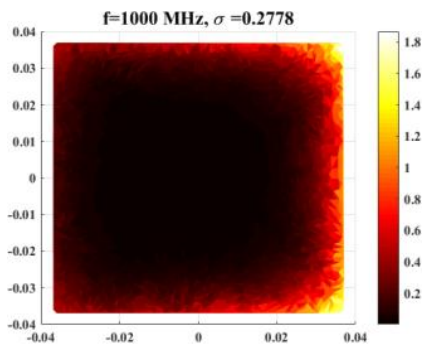

(e)

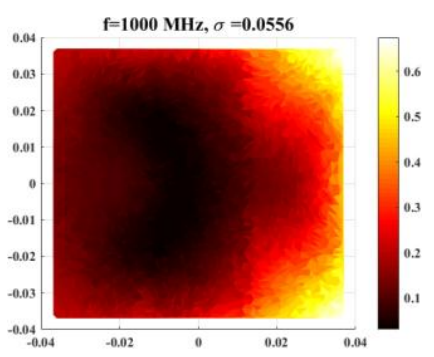

(d)

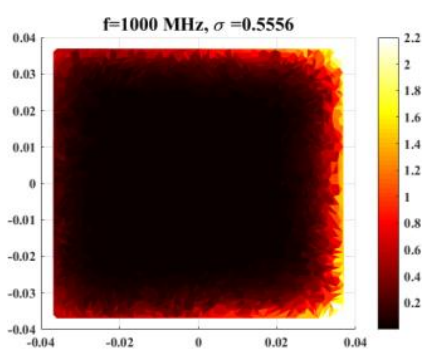

(f)

Figure 9: Distribution of fields in an inhomogeneous square scatterer illuminated by an incident field at $\mathbf{- x}$ direction with increasing $\sigma$

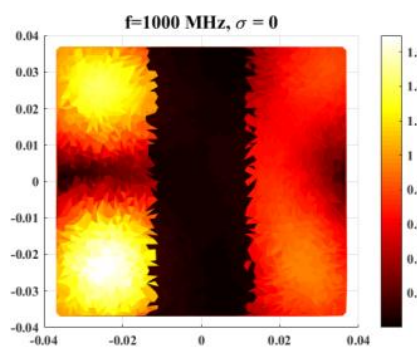

(a)

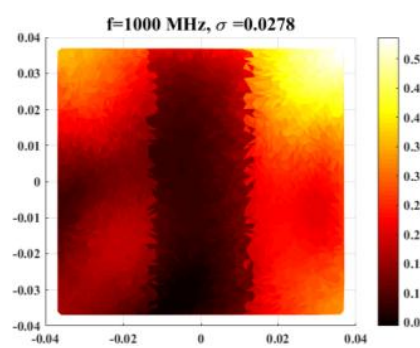

(c)

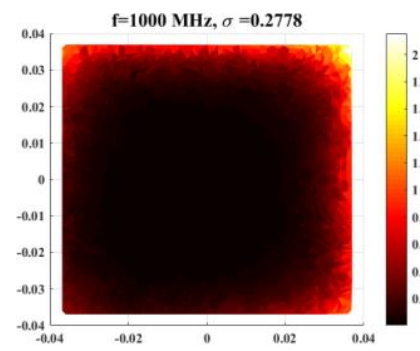

(e)

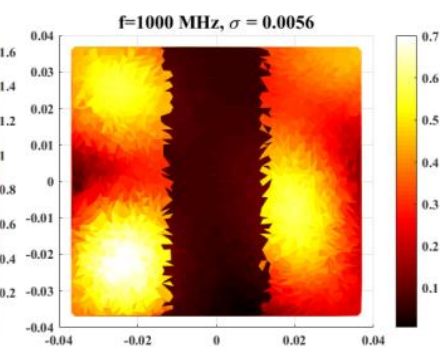

(b)

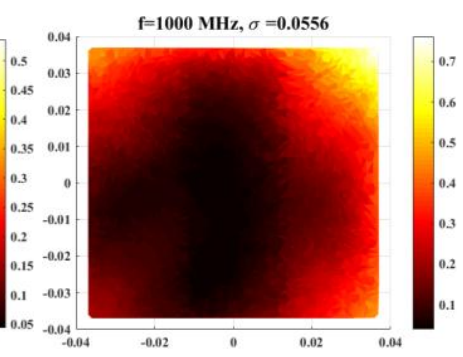

(d)

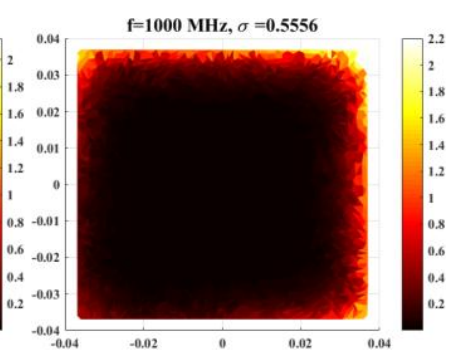

(f)

Figure 10: Distribution of fields in an inhomogeneous square scatterer illuminated by an incident field at 45 degrees to $-\mathbf{x}$ direction with increasing $\sigma$ 
3.5. Wideband study of a scatterer with constant electrical dimensions and constant conductivity

After demonstrating the physical phenomena on different structures with SFIE method, wideband studies, where SFIE is powerful are conducted. As mentioned before, the scatterer is discretized in volume and on surface. Volume integrals in different frequencies are related to each other with simple algebraic manipulations. Therefore, it is expected that SFIE solves the problem more efficiently. Efficiency, in that context, is used as a faster solution within a certain error limit. Frequency bandwidth is chosen to be between $100 \mathrm{MHz}$ and $1 \mathrm{GHz}$.

3.5.1. Change in RCS curves with frequency, conductivity and electrical dimensions

Earlier studies show that total RCS decreases with frequency [10]. Since frequency and wavelength are inversely proportional, decreasing frequency would make the scatterer electrically smaller. Then the scatterer acts like a point scatterer after some point. This also leads to flattened RCS curves.

In previous parts it is shown that increasing conductivity causes skin effect and flattens RCS curves, with decreasing maximum RCS value. Decrease in frequency contributes to this fact as shown in Figure 11.

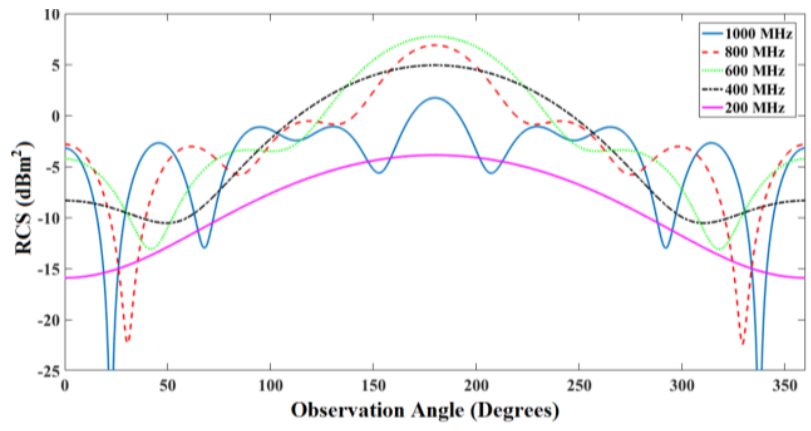

Figure 11: Wideband RCS results of a lossless scatterer.

These two observations show that high conductivity and low frequency would result in a flat RCS plot. This is shown in Figure 12 (a-f). The scatterer is chosen to be circular, homogeneous and it has a radius of $1 \lambda$.

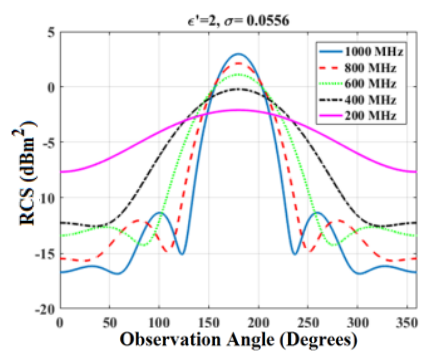

(a)

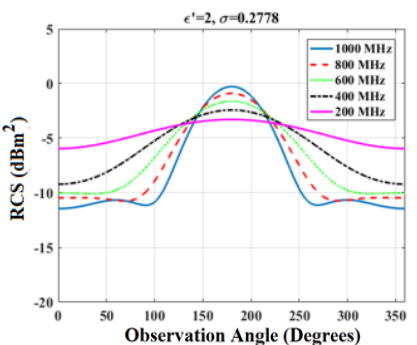

(b)

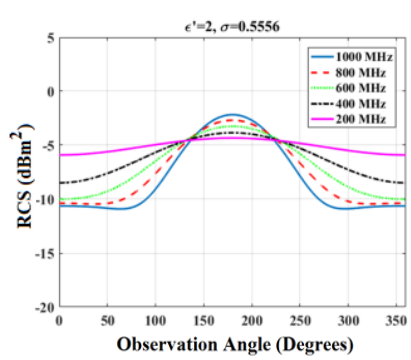

(c)

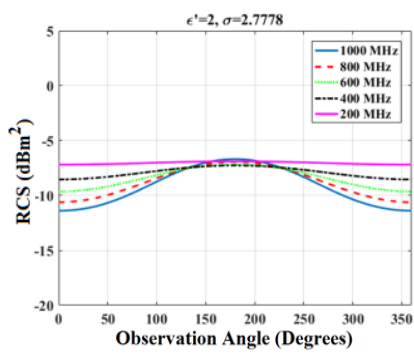

(e)

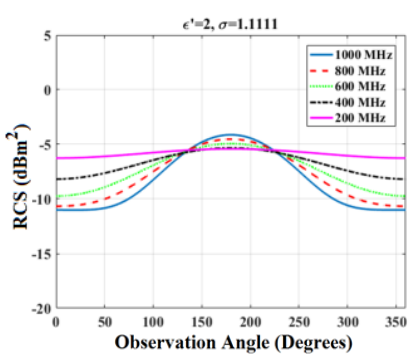

(d)

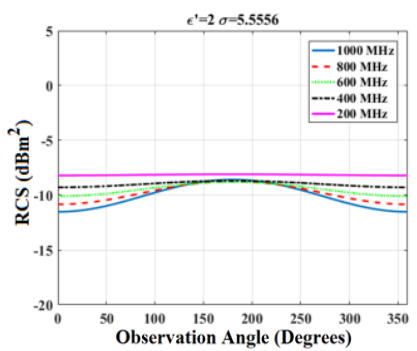

(f)
Figure 12: Wideband RCS results of scatterer while $\sigma$ changes. (a) $\sigma=0.0556$, (b) $\sigma=0.2778$, (c) $\sigma=0.5556$, (d) $\sigma=1.1111$, (e) $\sigma=2.7778$, (f) $\sigma=5.5556$.

To demonstrate that the flattening is related to the dimensions, the RCS plots for the largest $\sigma$ value are recalculated for bigger structures. Curvatures start to show again as radius increases as in Figure 13 (a-e).

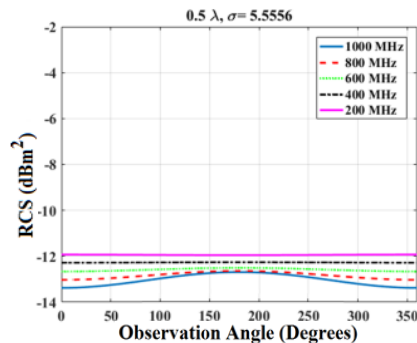

(a)

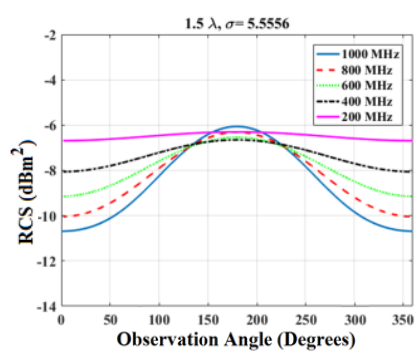

(c)

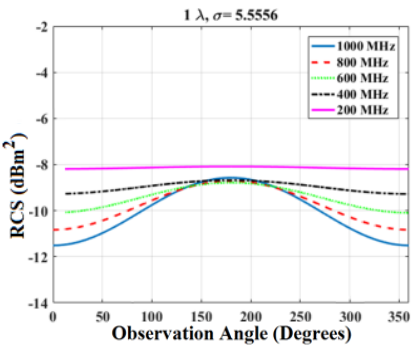

(b)

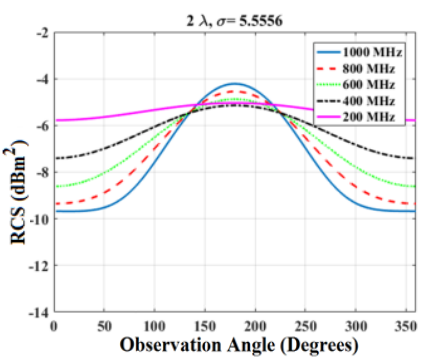

(d)

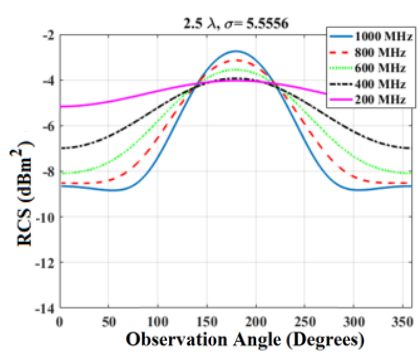

(e) 
Figure 13: Effect of physical dimensions on RCS of a highly conducting scatterer. (a) $\mathrm{r}=0.5 \lambda$, (b) $\mathrm{r}=1 \lambda$, (c) $\mathrm{r}=1.5 \lambda$, (d) $\mathrm{r}=2 \lambda$, (e) $\mathrm{r}=2.5 \lambda$.

3.5.2. Comparison of SFIE and MoM with respect to observation angle, frequency, discretization fineness and conductivity

After showing these physical facts with SFIE method, comparison of MoM and SFIE results are given. The differences between results of the two methods are given with respect to RCS observation angle and frequency. For compactness, numerical results of one case for each parameter will be shown here.

\section{1-Observation angle}

A circular and homogeneous scatterer with $1.5 \lambda$ radius is analyzed. Discretization is done with 1988 triangles and $\sigma$ is chosen to be 0.2778 . Initial frequency is set to be $1 \mathrm{GHz}$. The differences between MoM and SFIE results with respect to observation angle are shown for decreasing frequencies as in Figure 14.
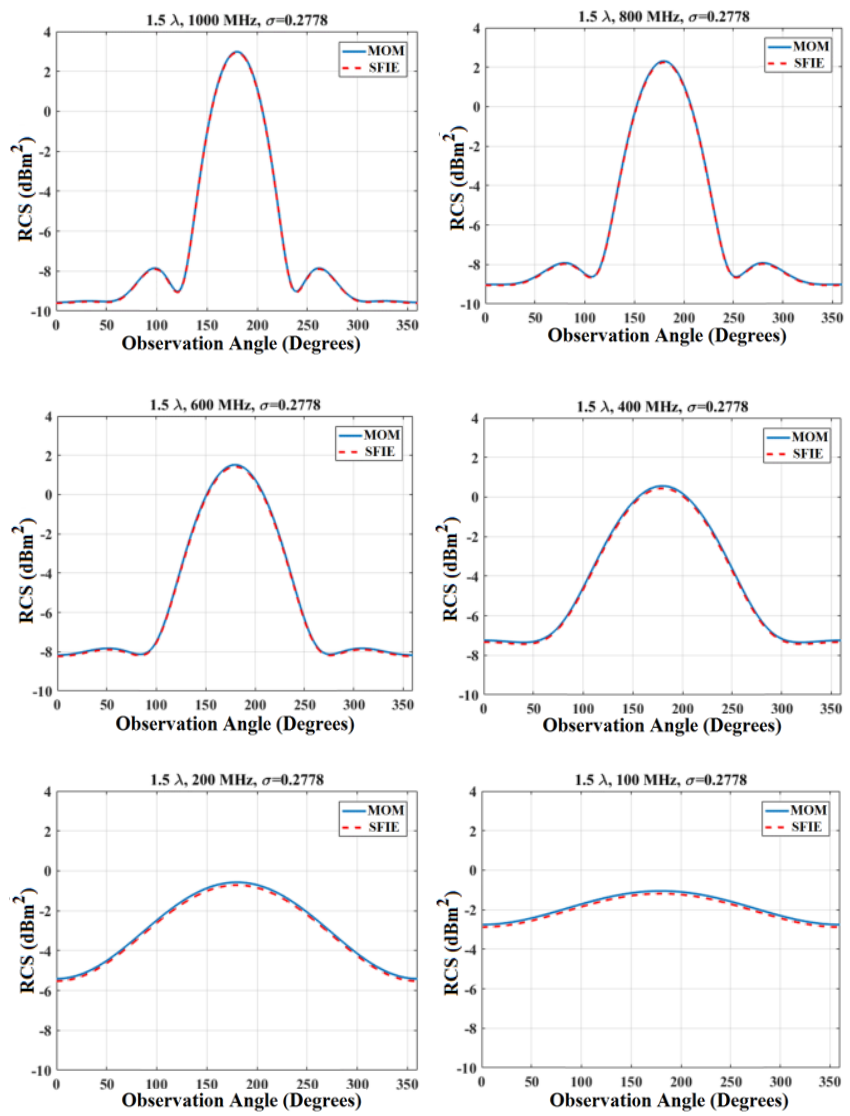

Figure 14: Comparison of RCS curves found by SFIE and MoM for $\mathrm{r}=1.5 \lambda$ and $\sigma=0.2778$ as frequency decreases

The results of MoM and SFIE for frequencies close to $\omega_{0}$ are very similar, but when the frequency shift increases or loss is high the RCS curves from MoM and SFIE start to differ slightly. This is consistent with the stated idea that electrical dimensions of the scatterer shrinks with these two parameters and the comparison becomes unhealthy for extreme cases.

\section{2-Frequency}

Differences between RCS results found by the two methods are shown in wideband in Figure 15 below. The effect of number of discretizing triangles can also be seen. Euclidean norm is used to find the difference. The scatterer has $1.5 \lambda$ radius and $\sigma$ is chosen to be $0,0.0556,0.2778,0.5556$, $1.1111,2.7778$ and 5.5556 as in previous comparison.

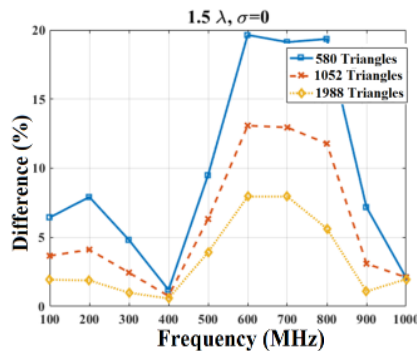

(a)

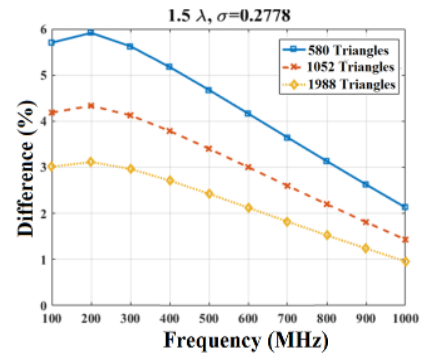

(c)

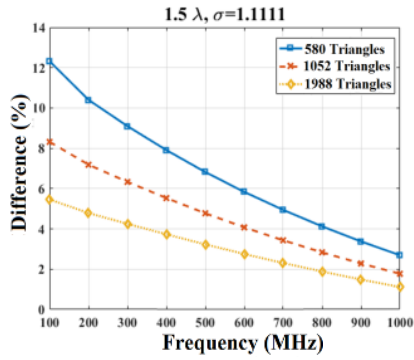

(e)

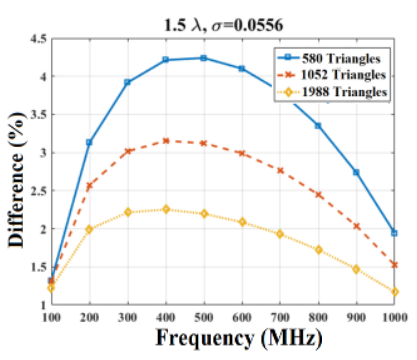

(b)

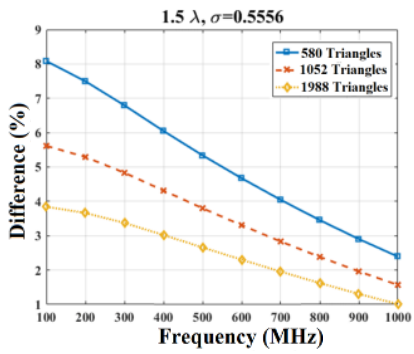

(d)

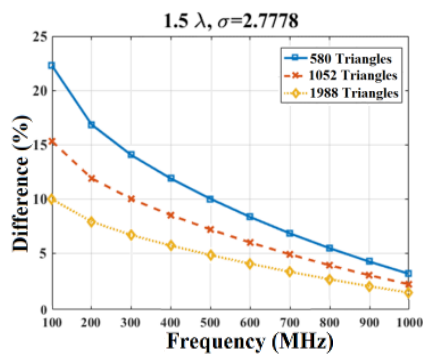

(f)
Figure 15: RCS difference percentage between MoM and SFIE in wideband for (a) $\sigma=0$ (b) $\sigma=0.0556$, (c) $\sigma=0.2778$, (d) $\sigma=0.5556$, (e) $\sigma=1.1111$, (f) $\sigma=2.7778$

Since RCS is a function of field distribution it is expected to see a similar set of results in fields comparison. However, it should be noted that the field comparison is made just for $\mathbf{E}_{\mathbf{Z}}$ field and RCS calculations require the $\mathbf{H}_{\mathbf{x}}$ and $\mathbf{H}_{\mathbf{y}}$ contributions too. That is why it is not expected to see exactly the same plots for RCS and field comparisons. 


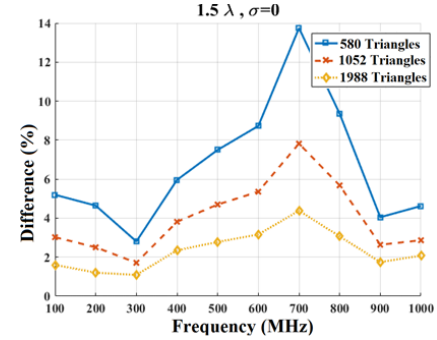

(a)

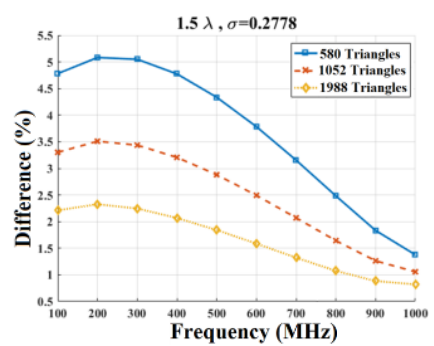

(c)

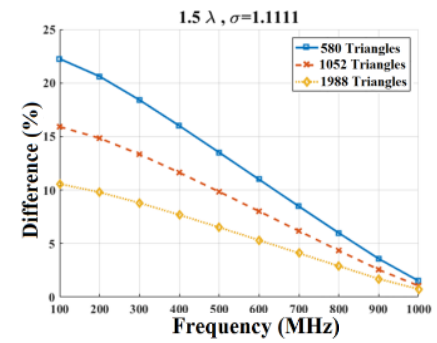

(e)

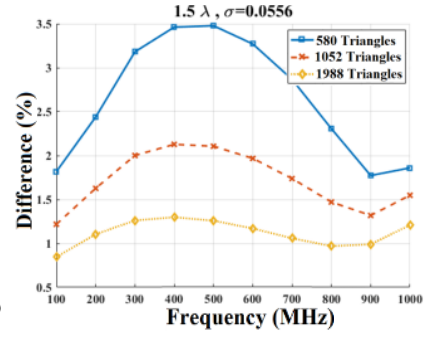

(b)

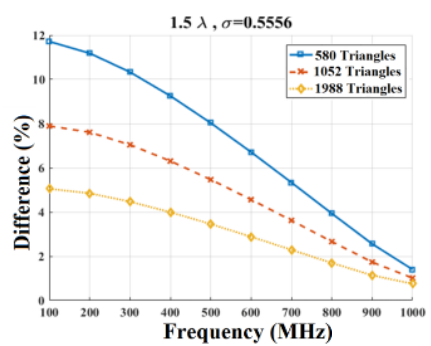

(d)

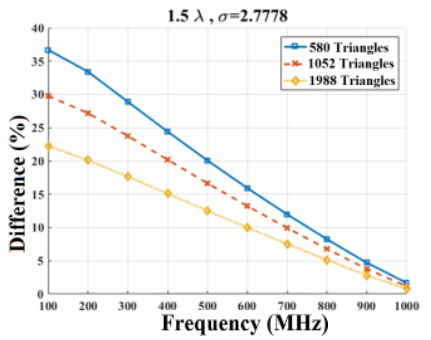

(f)
Figure 16: Field difference percentage between MoM and SFIE in wideband for (a) $\sigma=0$ (b) $\sigma=0.0556$, (c) $\sigma=0.2778$, (d) $\sigma=0.5556$, (e) $\sigma=1.1111$, (f) $\sigma=2.7778$

\section{Conclusions}

This work investigates in detail the application of Shifted Frequency Internal Equivalence (SFIE) method to two dimensional lossy objects. Increasing conductivity constant let skin effect to be seen better and shrank the object electrically as expected. Skin effect and shrinkage both flatten the RCS curves. An inhomogeneous object is investigated for a set of conductivities. The field strength between different pieces started to be smoother as loss increases. Also edge diffraction effect is observed. Finally, MoM and SFIE methods are compared in wideband to see the differences in RCS and field parameters with respect to observation angle and frequency.

It is concluded that when conductivity increases more than 0.5 , skin effect is dominant and fields are very close to the surface. This basically converts the volume problem to a surface problem. SFIE, by formulation, has both volume and surface unknowns and the number of volume elements are much more than that of surface elements. This leads to although small, some differences. Even under these circumstances, within a large bandwidth, the results from SFIE and MoM solutions are reasonably close to each other. In future work, surface MoM results for homogeneous scatterer will be compared with SFIE to validate the results.

\section{References}

[1] R. F. Harrington, Field Computation by Moment Methods, Macmillan, New York, US, 1968.

[2] J. Jin, The Finite Element Method in Electromagnetics, John Wiley \& Sons, New York, US, 2014.

[3] L. Zheng-liang, G.Shu-xi, M. Ji and W.Xing, "Impedance matrix interpolation based on method of moments for solving radiation problem over a frequency band", Int. Con. On Micr. And Millimeter Wave Tech., $3,1,1-3,2012$.

[4] V. V. S. Prakash and R. Mittra, "Characteristic basis function method: A new technique for efficient solution of method of moments matrix equations", Microw. And Opt. Tech., 36,2,95-100,2003.

[5] P. Bradley, C. Brennan and M. Condon, "Efficient Wideband Electromagnetic Scattering Computation for Frequency Dependent Lossy Dielectrics Using WCAWE", IEEE Tran. on Ant. and Prop, 57, 10, 2009.

[6] A. Ünal, S. Özdemir, A. Köksal, "A Direct Proof of SFIE", IEEE Trans. on Antennas and Propagation, Vol. 66, No. 4, pp. 2141-2142, Apr. 2018.

[7] A. Köksal, "Shifted-Frequency Internal Equivalence", IEEE Trans. Microw. Theory Tech., Vol. 46, No. 1, pp. 76-81, Jan. 1988.

[8] A. Köksal, "Multifrequency Formulation for Electromagnetic Scattering Using Shifted-Frequency Internal Equivalence", IEEE Trans. Microw. Theory Tech., Vol. 47, No. 2, pp. 150-155, Feb. 1999.

[9] S. Özdemir, A. Ünal, A. Köksal, “Application of Shifted Frequency Internal Equivalence to Multi-frequency Scattering Problems", IEEE Trans. on Antennas and Propagation, Vol. 65, 9, Sept. 2017.

[10]A. Ünal, A. Köksal, Kaydırılmış Frekansta İ̧̧ Eşdeğerlik Algoritmasının İki Boyutlu Elektromanyetik Saçılma Problemlerinde Başarımının Incelenmesi, Hacettepe University, Electrical and Electronics Engineering, MSc. Thesis, 2016.

[11]D.K. Cheng, Fundamentals of Engineering Electromagnetics, Pearson, 1992.

[12]A. Ishimaru, Electromagnetic Wave Propagation, Radiation, and Scattering, Prentice Hall, 1991.

[13] R., Curiac, I.R., Ciric, "Analysis of Wave Scattering by a Lossy Dielectric Using Single Source Surface Integral Equations", IEEE CCECE. Canadian Conference on Electrical and Computer Engineering. Conference Proceedings, 2002. 